\title{
DIALELO PARCIAL ENTRE CLONES DE Eucalyptus camaldulensis E CLONES DE E. urophylla, E. grandis E E. saligna ${ }^{1}$
}

\author{
Odair Bison², Magno Antônio Patto Ramalho ${ }^{3}$, Gabriel Dehon S. P. Rezende ${ }^{4}$, Aurélio Mendes Aguiar e $^{5}$ \\ Marcos Deon Vilela de Resende ${ }^{6}$
}

\begin{abstract}
RESUMO - O objetivo deste trabalho foi identificar combinações híbridas superiores nos cruzamentos entre clones-elite de Eucalyptus grandis, E. urophylla e E. saligna com clones-elite de E. camaldulensis da Aracruz Celulose S.A., por meio de cruzamentos dialélicos parciais. Para isso, as 44 combinações híbridas obtidas e mais quatro testemunhas foram plantadas de setembro a outubro de 2001, em três localidades: Aracruz, ES; São Mateus, ES; e Caravelas, BA, no delineamento de blocos casualizados com 40 repetições e uma planta por parcela. Dois anos após o plantio, foram avaliadas a circunferência à altura do peito (CAP) e a densidade básica da madeira (DEN). Os dados médios foram submetidos à análise dialélica segundo o método de Griffing (1956), adaptado por Geraldi e Miranda Filho (1988). Constatou-se que muitos híbridos foram promissores tanto para a obtenção de ganhos em CAP quanto para obter a densidade da madeira, pois associaram média e variância altas. A maior parte da variação entre os híbridos foi explicada pela capacidade geral de combinação, indicando a predominância de efeitos aditivos no controle dos caracteres.
\end{abstract}

Palavras-chave: Eucalipto, melhoramento e capacidade de combinação.

\section{HALF-DIALLEL BETWEEN Eucalyptus camaldulensis CLONES AND E. grandis E. urophylla, AND E. saligna CLONES}

\begin{abstract}
The objective of this research was to evaluate the hybrid potential between $\boldsymbol{E}$. grandis, $\boldsymbol{E}$. urophylla and $\boldsymbol{E}$. saligna clones from Aracruz Celulose $S$. A. with $\boldsymbol{E}$. camaldulensis clones. To do so, six elite clones were crossed with ten $\boldsymbol{E}$. camaldulensis clones, in a half-diallel mating design. The resulting hybrid combinations as well as the four check clones were carried out from September to October 2001, in three sites, Aracruz and São Mateus, in the Espírito Santo State, and Caravelas, Bahia State, in a completely randomized block design with single plant plots and 40 replicates. Two years later, the circumference at breast height and the wood basic density were measured. The means were submitted to a diallel analysis, according to the Griffing (1956) method, adapted by Geraldi and Miranda Filho (1988). It was observed that the diallel crossings were promising for both the wood volume and wood quality gain. The general combining ability was responsible for most of the variations between the hybrids.
\end{abstract}

Keywords: Eucalyptus, breeding and combining ability.

\footnotetext{
${ }^{1}$ Recebido em 04.07.2007 e aceito para publicação em 24.04.2009.

${ }^{2}$ Centro de Tecnologia Canavieira, Variedades. Faz. Santo Antônio s/n. Santo Antônio 13400-970 - Piracicaba, SP.

${ }^{3}$ Departamento de Biologia da Biologia. E-mail: <magnoapr@ufla.br>.

${ }^{4}$ RAIZ - Instituto de Investigação da Floresta e Papel, Aveiro, Portugal. E-mail: <gabriel.dehon@ @ortucelsoporcel.com>.

${ }^{5}$ Aracruz Celulose S.A. Rua São Geraldo 1800, Ermo 92500-000 - Guaiba, RS - Brasil. E-mail: <amendes@ aracruz.com.br>.

${ }^{3}$ Empresa Brasileira de Pesquisa Agropecuária, Centro Nacional de Pesquisa de Florestas. Colombo, PR. E-mail: <deon@ @npf.embrapa.br>.
} 


\section{INTRODUÇÃO}

No melhoramento de qualquer espécie, o importante é obter populações segregantes que associem média alta com a maior variância possível. Para identificar populações segregantes que associem esses dois atributos há algumas metodologias. Uma das mais utilizadas são os cruzamentos dialélicos que, além de permitir identificar as populações segregantes mais promissoras, possibilitam obter informações a respeito do controle genético dos caracteres (CRUZ et al., 2004).

No caso do Eucalyptus, visando à produção de celulose no Brasil, os trabalhos de seleção têm se concentrado em populações e, ou, clones das espécies E. grandis e E. urophylla, ou híbridos entre elas. Essas duas espécies são bem adaptadas e têm possibilitado ganhos expressivos em volume de madeira (VENCOVSKY e RAMALHO, 2000; GONÇALVES et al., 2001). Contudo, é importante a introgressão de alelos de outras espécies visando ampliar a base genética e também possibilitar o melhoramento para outros caracteres, além do volume. Uma das opções é o cruzamento dos melhores clones utilizados pelas empresas de celulose com clones superiores de E. camaldulensis, que também é adaptado às condições de cultivo prevalecentes no Brasil e associa bom volume de madeira com alta densidade.

Informações a respeito do controle genético de caracteres do gênero Eucalyptus no Brasil têm sido obtidas em várias oportunidades, utilizando progênies de meios-irmãos (PAULA et al., 1996; GONÇALVES et al., 1997). Contudo, são escassas as informações a respeito da capacidade de combinação que possam orientar os futuros trabalhos de melhoramento envolvendo hibridações, sobretudo entre clones.

Os objetivos deste trabalho foram identificar combinações híbridas superiores nos cruzamentos entre clones-elite de E. grandis, E. urophylla e E. saligna com clones-elite de E. camaldulensis e verificar qual o tipo de interação alélica predominante na expressão da circunferência à altura do peito e densidade da madeira.

\section{MATERIAL E MÉTODOS}

Foram avaliadas combinações híbridas provenientes de um dialelo parcial entre seis clones-elite de eucalipto da Aracruz Celulose S.A. (Tabela 1) e 10 clones de E. camaldulensis da V \& M Florestal Ltda. Das 60 combinações possíveis, foram obtidas e avaliadas 44 . Para a realização dos cruzamentos, pólen de 10 clones superiores de E. camaldulensis da Empresa V \& M Florestal Ltda. foi enviado para a Aracruz Celulose S.A. Desse modo, a propriedade desses clones ficou preservada pela empresa detentora deles. As sementes híbridas foram obtidas em cruzamentos realizados na área experimental da empresa Aracruz Celulose S.A., as quais foram coletadas e semeadas em tubetes na casa de vegetação para a obtenção das mudas. Também foram produzidas mudas dos quatro clones utilizados como testemunhas, os de números 1 a 4 (Tabela 1).

Os dialelos foram avaliados em três locais de plantio comerciais da empresa Aracruz Celulose S.A., que foram Aracruz, ES, 19 50' 19" de latitude S e 40'12' 43" de longitude W, São Mateus, ES, 18 36' 13"' de latitude $\mathrm{S}$ e $40^{\circ} 01$ ' 32 " de longitude W e Caravelas, BA, $17^{\circ}$ 47' 17" de latitude S e 39 33' 57" de longitude W. O experimento foi instalado de setembro a outubro de 2001, adotando-se o delineamento de blocos casualizados com 40 repetições e uma planta por parcela, com espaçamento entre plantas de 3 x $3 \mathrm{~m}$. Os clones de números 1 a 4 foram incluídos como testemunhas nos três experimentos. O manejo dos experimentos foi o mesmo adotado no plantio comercial.

Dois anos após a implantação dos experimentos foram tomados os seguintes dados:

- Circunferência à altura do peito $(\mathrm{cm})$; e

Tabela 1 - Relação dos clones-elite utilizados nos cruzamentos com E. camaldulensis

Table 1 - List of the elite clones used in the crossings with E. camaldulensis

\begin{tabular}{cl}
\hline Clones-elite & \multicolumn{1}{c}{ Origem } \\
\hline 1 & Híbrido cuja mãe é de E. grandis, de Rio Claro, SP \\
2 & Híbrido cuja mãe é de E. saligna, de Rio Claro, SP \\
3 & Híbrido cuja mãe é de E. urophylla, de Rio Claro, SP \\
4 & Híbrido cuja mãe é de E. grandis, de Rio Claro, SP \\
5 & Híbrido de E. urophylla, de Flores-Indonésia \\
6 & Híbrido de E. grandis, de Zimbabwe \\
\hline
\end{tabular}

R. Árvore, Viçosa-MG, v.33, n.3, p.395-402, 2009 
- Penetração da agulha do pilodyn na madeira ( $\mathrm{mm})$.

A partir da avaliação realizada com o pilodyn, foi estimada a densidade básica da madeira $\left(\mathrm{kg} / \mathrm{m}^{3}\right)$, pela expressão: Densidade $=615$ - $11 *$ Leitura do pilodyn. Para a obtenção dessa expressão, anteriormente foram avaliadas 142 árvores de diferentes espécies de eucalipto, com dois anos de idade, utilizando-se o pilodyn, e, em seguida, determinou-se a densidade básica da madeira no laboratório. A partir dessas avaliações foi construída a equação de regressão entre a leitura do pilodyn e a densidade básica da madeira.

Foi realizada, inicialmente, a análise de variância por local, em cada experimento e, posteriormente, a análise de variância conjunta. As análises de variância foram feitas empregando-se o Procedure for General Linear Models (PROC GLM) do SAS ${ }^{\circledR}$ (SAS, 2000).

Utilizando-se os dados médios por local e na análise conjunta, foram obtidas as estimativas da capacidade de combinação, usando o modelo de Griffing (1956), adaptado aos cruzamentos dialélicos parciais por Geraldi e Miranda Filho (1988). Considerando que nem todas as combinações foram obtidas e que houve diferenças no número de repetições, as análises dialélicas foram realizadas pelo método dos quadrados mínimos, utilizando-se o Procedure for Interactive Matrix Language (PROC IML) do SAS ${ }^{\circledR}$ (SAS, 2000).

\section{RESULTADOS E DISCUSSÃO}

Dos seis clones-elite utilizados nos cruzamentos com E. camaldulensis, dois de número 5 e 6 são híbridos interespecíficos e quatro devem ser híbridos naturais, em que se conhece apenas a espécie materna, duas de E. grandis (clones 1 e 4), uma E. saligna (clone 2) e uma de E. urophylla (clone 3). Provavelmente, as plantas que originaram esses clones são híbridos interespecíficos devido ao grande número de espécies introduzidos no Horto Florestal de Rio Claro, SP, e a possibilidade de ocorrência de cruzamento entre eles.

Nas análises conjuntas, as interações envolvendo híbridos e testemunhas $\mathrm{x}$ locais foram significativas ( $\mathrm{P}<0,01)$. Contudo, a contribuição da interação para a variação total foi pequena com relação a ambos os caracteres avaliados. Para a circunferência à altura do peito (CAP), por exemplo, a soma de quadrados (SQ) da interação foi de apenas $17 \%$ da SQ dos tratamentos. Constatou-se também que embora fosse detectada diferença significativa $(\mathrm{P}<0,01)$ entre locais, $\mathrm{O}$ desempenho médio variou pouco entre eles (Tabela 2). Dessa forma, os resultados serão apresentados com ênfase na média dos três locais.

O desempenho médio dos híbridos foi 4,1\% inferior ao da média das testemunhas para a CAP. No caso da densidade básica da madeira (DEN), a média dos híbridos foi $1 \%$ superior à média das testemunhas (Tabela 2). Tomando como referência o clone 4 , o mais utilizado pela empresa, verificou-se para a CAP que o seu desempenho médio foi superior ao dos demais clonestestemunha avaliados (Tabela 3 ). Veja, contudo, que três dos híbridos $(6 \times 1,6 \times 2$ e $2 \times 7)$ ficaram no mesmo grupo do clone 4 . Esse fato é mais expressivo quando se considera que o desempenho dos híbridos é proveniente da média de 40 plantas em cada local e que, como a variação genética entre elas é grande, é provável que novos clones com volume de madeira superior ao do clone 4 possam ser obtidos.

Tabela 2 - Média dos híbridos e testemunhas para a circunferência à altura do peito (CAP) e a densidade básica da madeira (DEN), obtidas na avaliação de cruzamentos dialélicos entre clones de E. camaldulensis e clones-elite aos 2 anos de idade

Table 2 - Means of the hybrids and controls for the circumference at breast height (CAP) and basic wood density (DEN) obtained in the evaluation of diallel crossings between two-year-old elite clones and E. camaldulensis

\begin{tabular}{|c|c|c|c|c|c|}
\hline \multirow[t]{2}{*}{ Caractere } & & \multicolumn{4}{|c|}{ Locais } \\
\hline & & Aracruz & São Mateus & Caravelas & Conjunta \\
\hline \multirow{4}{*}{ CAP $(\mathrm{cm})$} & Média dos híbridos & 28,99 & 29,81 & 31,20 & 30,00 \\
\hline & Média das testemunhas & 29,06 & 31,74 & 33,06 & 31,29 \\
\hline & Média geral & 29,07 & 30,31 & 31,47 & 30,28 \\
\hline & $\mathrm{CV} \%$ & 16,46 & 20,69 & 19,36 & 18,80 \\
\hline \multirow{4}{*}{$\operatorname{DEN}\left(\mathrm{k} / \mathrm{m}^{3}\right)$} & Média dos híbridos & 446,11 & 438,56 & 422,07 & 435,58 \\
\hline & Média das testemunhas & 443,34 & 436,60 & 408,53 & 429,49 \\
\hline & Média geral & 445,91 & 437,48 & 420,76 & 434,35 \\
\hline & $\mathrm{CV} \%$ & 2,99 & 3,30 & 3,65 & 3,32 \\
\hline
\end{tabular}




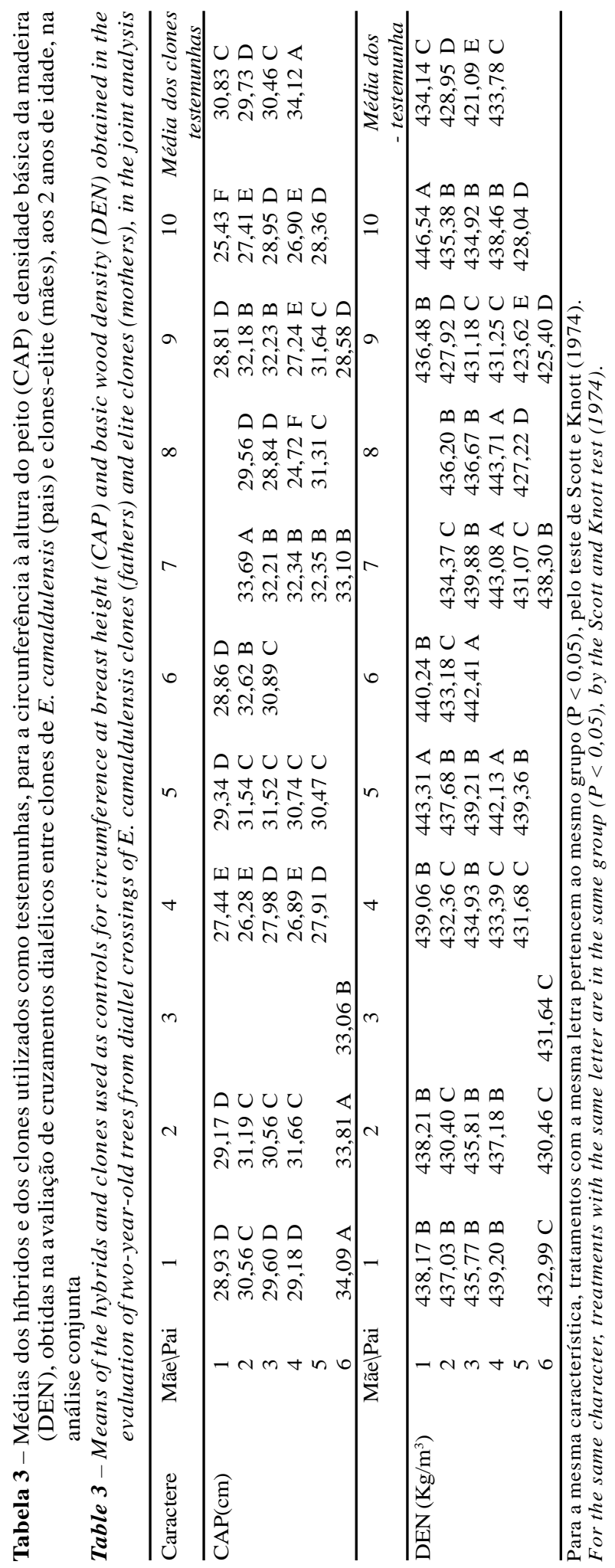

No caso da Aracruz Celulose S.A., a faixa de densidade básica da madeira adotada atualmente é de 500,0 a $550,0 \mathrm{~kg} / \mathrm{m}^{3}$. Tomando como referência novamente o clone 4, a densidade básica desse clone, aos 7 anos de idade, é de $575,0 \mathrm{~kg} / \mathrm{m}^{3}$ (AGUIAR, 2004) ${ }^{1}$. Verificase que, aos dois 2 de idade, o clone 4 apresentou densidade inferior à obtida na idade de corte das árvores (Tabela 3). Contudo, como a densidade apresenta ligeiro aumento com a idade, é interessante comparar a densidade básica dos híbridos obtidos com a desse clone. Chama-se a atenção o fato de que $61,3 \%$ dos híbridos (27 combinações) apresentaram desempenho médio superior ao do clone 4 .

O resultado da análise de variância do dialelo indica que o desempenho dos híbridos dependeu tanto da capacidade geral de combinação (CGC) dos genitores do grupo 1 e grupo 2 quando da capacidade específica de combinação (CEC). Isso porque, em todos os casos, o teste de $\mathrm{F}$ foi significativo $(\mathrm{P}<0,01)$ para essas fontes de variação (Tabela 4). Embora ocorressem diferenças significativas em todos os casos, a contribuição relativa da soma de quadrados dessas fontes de variação para a variação total foi diferente. Para a CAP, constatase que $72,9 \%$ da soma de quadrados atribuída aos híbridos foram explicadas pela CGC e apenas $27,1 \%$ pela CEC. Para a DEN, a contribuição da CGC $(81,5 \%)$ também foi bem superior à da CEC $(18,5 \%)$.

A capacidade geral de combinação de um parental corresponde ao desvio de seu desempenho médio em combinações híbridas. Quando existe predominância de CGC, isso indica que no controle do caráter há predominância dos efeitos aditivos. Já a CEC é estimada como desvio do comportamento em relação ao que seria esperado com base na CGC. Assim, ela avalia a contribuição dos efeitos não aditivos, dominância ou epistasia, na expressão dos caracteres (CRUZ et al., 2004). Portanto, em princípio, os resultados possibilitaram inferir que no controle genético desses caracteres há predominância de efeitos aditivos, embora a dominância também seja importante. A ocorrência de efeitos aditivos na expressão de caracteres de Eucalyptus é frequente na literatura (PAULA et al., 1996). A presença de dominância também tem sido relatada

${ }^{1}$ AGUIAR, A.M. Comunicação pessoal. 2004. (Aracruz Celulose S.A., Centro de Pesquisa e Tecnologia, 29.197-900 Aracruz, Espírito Santo, Brasil). 
em algumas oportunidades. A estimativa do grau médio de dominância obtido no cruzamento entre E. grandis $\mathrm{x}$ E. urophylla, para o caráter volume de madeira, aos 3 anos de idade, foi de 0,6 (REZENDE e RESENDE, 2000). Resultados semelhantes foram relatados sobre o híbridoE. urophylla x E. pellita (BOUVETe VIGNERON, 1996). Em híbridos envolvendo progênies de E. grandis $\mathrm{x}$ E. urophylla foi constatado que a heterose média para CAP foi de 38,7\% (BISON et al., 2006). Isso comprova que a variância de dominância contribui para a manifestação do caráter volume de madeira. Já para a densidade da madeira os resultados encontrados na literatura indicam que a variância de dominância tem menor importância na manifestação dessa característica (ASSIS, 2000). Esses resultados concordam com os obtidos neste trabalho. Resultados semelhantes foram obtidos por Bison et al. (2007) em um dialelo parcial, conduzido nos mesmos locais, envolvendo os mesmos clones-elite com clones de E. globulus.

As estimativas da capacidade de combinação nos cruzamentos dialélicos entre clones de E. camaldulensis x clones-elite, para a CAP e a DEN, são apresentadas na Tabela 5. Para a CAP, observou-se, no grupo 1 (cloneselite), que os clones com estimativa da CGC positiva foram os de números 2, 5 e 6 (Tabela 5). A princípio, era esperado que o clone 4 apresentasse CGC positiva, pois ele possuía média alta. Provavelmente, esse clone é vigoroso devido à ocorrência da maioria dos locos em heterozigose e, ou, a presença de combinações epistáticas favoráveis que são desfeitas por ocasião do cruzamento. Esse fato é justificado pela sua grande depressão quando autofecundado (BISON et al., 2004). É esperado que os clones com CGC positiva devem ter maior frequência de locos com alelos favoráveis em homozigose. Desse modo, fica fácil explicar a menor estimativa da CGC do clone 4 para a CAP. No grupo 2 (E. camaldulensis), os clones com CGC positiva foram os de números 2, 5, 6 e 7. Vale salientar que o clone 3 participou de apenas uma combinação híbrida, e, portanto, a sua estimativa de CGC, embora positiva, não é comparável às demais.

Considerando-se apenas um loco, a capacidade de combinação (CC) é obtida pela expressão: $C C=\left(p_{i}-\bar{p}\right)[\alpha+(1-2 \bar{r}) \delta]$, em que $\mathrm{p}_{\mathrm{i}}$ é a frequência do alelo favorável para o referido loco no genitor sob avaliação; $\bar{p}$ é a frequência alélica média dos genitores do mesmo grupo; $\bar{r}$ é a frequência alélica média dos genitores do outro grupo; $\alpha$ é a contribuição do loco em homozigose em relação à média $\mathrm{e} \delta$ é a contribuição do heterozigoto em relação à média. A capacidade de combinação depende, assim, da contribuição dos efeitos aditivos $(\alpha)$ e de dominância $(\delta)$. Neste último caso, a contribuição será possível se o testador (genitor do outro grupo) apresentar frequência alélica média inferior a 0,5 (VENCOVSKY, 1987). No caso da circunferência à altura do peito, maior variação da CGC ocorreu entre os clones do grupo 2, E. camaldulensis, ou seja, no grupo com menor frequência de alelos favoráveis para o referido caráter. O contrário foi observado com relação à densidade básica da madeira.

Já a capacidade específica de combinação (CEC) é obtida pela expressão: $C E C=2\left[\left(\bar{p}-p_{i}\right)\left(r_{i}-\bar{r}\right)\right] \delta$ (VENCOVSKY, 1987).Assim, a CEC depende da divergência entre os genitores e da ocorrência de dominância. Como os genitores pertencem a "pools" gênicos diferentes e também são de origens diferentes, infere-se que a CEC foi de menor magnitude devido à pequena contribuição da dominância na expressão dos caracteres, especialmente para a densidade básica da madeira.

Tabela 4 - Resumo das análises de variância do dialelo parcial, considerando-se a média dos três locais, para a circunferência à altura do peito (CAP) e a densidade básica da madeira (DEN), obtidas na avaliação de cruzamentos entre clones de E. camaldulensis e clones-elite, aos 2 anos de idade

Table 4-Summary of the analyses of variance of the half-diallel, considering the mean of the three places for circumference at breast height (CAP) and basic wood density (DEN) obtained in the evaluation of two-year-old trees from crossings between E. camaldulensis and elite clones

\begin{tabular}{|c|c|c|c|c|c|c|c|}
\hline \multirow[b]{2}{*}{$\mathrm{FV}^{1}$} & \multirow[b]{2}{*}{ GL } & \multicolumn{3}{|c|}{$\mathrm{CAP}(\mathrm{cm})$} & \multicolumn{3}{|c|}{$\operatorname{DEN}\left(\mathrm{kg} / \mathrm{m}^{3}\right)$} \\
\hline & & SQ & $\mathrm{QM}$ & $\mathrm{P}$ & SQ & $\mathrm{QM}$ & $\mathrm{P}$ \\
\hline$\overline{\mathrm{CGC} 1}$ & 5 & 4010,35 & 802,07 & 0,01 & 47492,55 & 9488,51 & 0,01 \\
\hline CGC2 & 9 & 10598,13 & 1177,57 & 0,01 & 39760,65 & 4417,85 & 0,01 \\
\hline CEC & 29 & 5436,34 & 187,46 & 0,01 & 19744,07 & 680,83 & 0,01 \\
\hline Erro & 4473 & 145059,38 & 32,43 & & 928907,91 & 207,67 & \\
\hline
\end{tabular}

1/ CGC1 - Capacidade geral de combinação dos clones de E. grandis e E. urophylla. CGC2 - Capacidade geral de combinação dos clones de E. camaldulensis.

${ }^{1 /}$ CGC1 - General combining ability for the E. grandis and E. urophylla clones; CGC2-General combining ability for the E. camaldulensis clones. 
Tabela 5 - Estimativa dos efeitos da capacidade geral de combinação do grupo 1 (CGC $)$, capacidade geral de combinação do grupo 2 (CGC $)$ e capacidade específica de combinação $\left(\mathrm{CEC}_{\mathrm{i}, \mathrm{j}}\right)$, para a circunferência à altura do peito (CAP) e densidade básica da madeira (DEN), obtidas na avaliação de cruzamentos dialélicos entre clones de E. camaldulensis (pais) e clones-elite (mães), aos 2 anos de idade, na análise conjunta

Table 5 - Estimate of the effects of the general combining ability of group 1 (CGC), general combining ability of group 2 (CGC) and specific combining ability $\left(C E C_{i j}\right)$, for the circumference at breast height (CAP) and basic wood density (DEN) obtained in the evaluation of two-year-old trees from diallel crossings of E. camaldulensis clones (fathers) and elite clones (mothers) in the joint analysis

\begin{tabular}{|c|c|c|c|c|c|c|c|c|c|c|c|c|}
\hline Caractere & MãelPai & 1 & 2 & 3 & 4 & 5 & 6 & 7 & 8 & 9 & 10 & $\mathrm{CGC}_{\mathrm{i}}$ \\
\hline \multirow{7}{*}{$\overline{\mathrm{CAP}(\mathrm{cm})}$} & 1 & $-0,044^{1 / \prime}$ & $-0,62$ & & $1,38^{*}$ & 0,00 & $-0,61$ & & & 0,27 & $-0,62$ & $-1,62^{*}$ \\
\hline & 2 & 0,67 & $-0,86$ & & $-2,04 *$ & $-0,06$ & $0,89^{*}$ & 0,77 & 0,36 & $1,38 *$ & $-0,90$ & $0,64 *$ \\
\hline & 3 & $-1,24 *$ & $-1,11^{*}$ & & 0,04 & 0,31 & $-0,46$ & $-0,32$ & 0,02 & $1,81 *$ & $1,03^{*}$ & 0,26 \\
\hline & 4 & 0,22 & $1,44 *$ & & 0,40 & $0,97 *$ & & $1,25^{*}$ & $-2,65^{*}$ & $-1,73 *$ & 0,43 & $-1,19 *$ \\
\hline & 5 & & & & $-0,58$ & $-1,29 *$ & & $-0,73^{*}$ & $1,95^{*}$ & 0,67 & $-0,11$ & $0,80 *$ \\
\hline & 6 & $2,10 *$ & $0.99 *$ & $!$ & & & & -0.58 & & $-2,99 *$ & & $1.40 *$ \\
\hline & $\mathrm{CGC}_{\mathrm{i}}$ & 0,47 & $1,29^{*}$ & $1,54 *$ & $-2,43^{*}$ & $0,84 *$ & $0,97 *$ & $2,16^{*}$ & $-1,56^{*}$ & 0,04 & $-2,45^{*}$ & \\
\hline \multirow[t]{8}{*}{$\overline{\operatorname{DEN}}\left(\mathrm{Kg} / \mathrm{m}^{3}\right)$} & Mãe\Pai & 1 & 2 & 3 & 4 & 5 & 6 & 7 & 8 & 9 & 10 & $\mathrm{CGC}_{\mathrm{i}}$ \\
\hline & $T$ & $-2,70 *$ & $-0,46$ & & $-0,08$ & $-1,87$ & $-2,38$ & & & 1,75 & 4,97 & $5,15 *$ \\
\hline & 2 & $3,32 *$ & $-1,11$ & & 0,38 & $-0,34$ & $-2,28 *$ & $-2,05$ & 1,41 & 0,35 & 0,97 & $-2,01 *$ \\
\hline & 3 & $-0,98 \stackrel{11}{1}$ & 1,26 & & $-0,08$ & $-1,84$ & $3,91 *$ & 0,42 & $-1,16$ & 0,57 & $-2,52 *$ & $1,02 *$ \\
\hline & 4 & 0,59 & 0,77 & & $-3,48 *$ & $-0,79$ & & 1,76 & $4,02 *$ & $-1,22$ & $-0,85$ & $2,88 *$ \\
\hline & 5 & & & & $3,44 *$ & $5,07 *$ & & $-1,62$ & $-3,84 *$ & $-0,22$ & $-2,64 *$ & $-5,74 *$ \\
\hline & 6 & $-0,19$ & $-0,52$ & $!$ & & & & $2,41 *$ & & $-1,64$ & & $-2,55 *$ \\
\hline & $\overline{C G C}$ & 0,21 & $-1,99 *$ & $-1,33$ & $-1,52 *$ & $4,52 *$ & $1,96 *$ & $2,92 *$ & 1,29 & $-5,93 *$ & 0,91 & \\
\hline
\end{tabular}

$\stackrel{\text { If }}{ } \mathrm{CEC}_{\mathrm{ij}}$ - parte interna da tabela; * - significativo a $5 \%$ de probabilidade, pelo teste $\mathrm{t} ;$ ! - $\mathrm{CEC}_{\mathrm{ij}}$ não estimada.

$\underline{I}$ CEC $C_{i j}$ - into the table; * - significant at $5 \%$ of probability, by the t test; ! - CEC $C_{i j}$ not estimated.
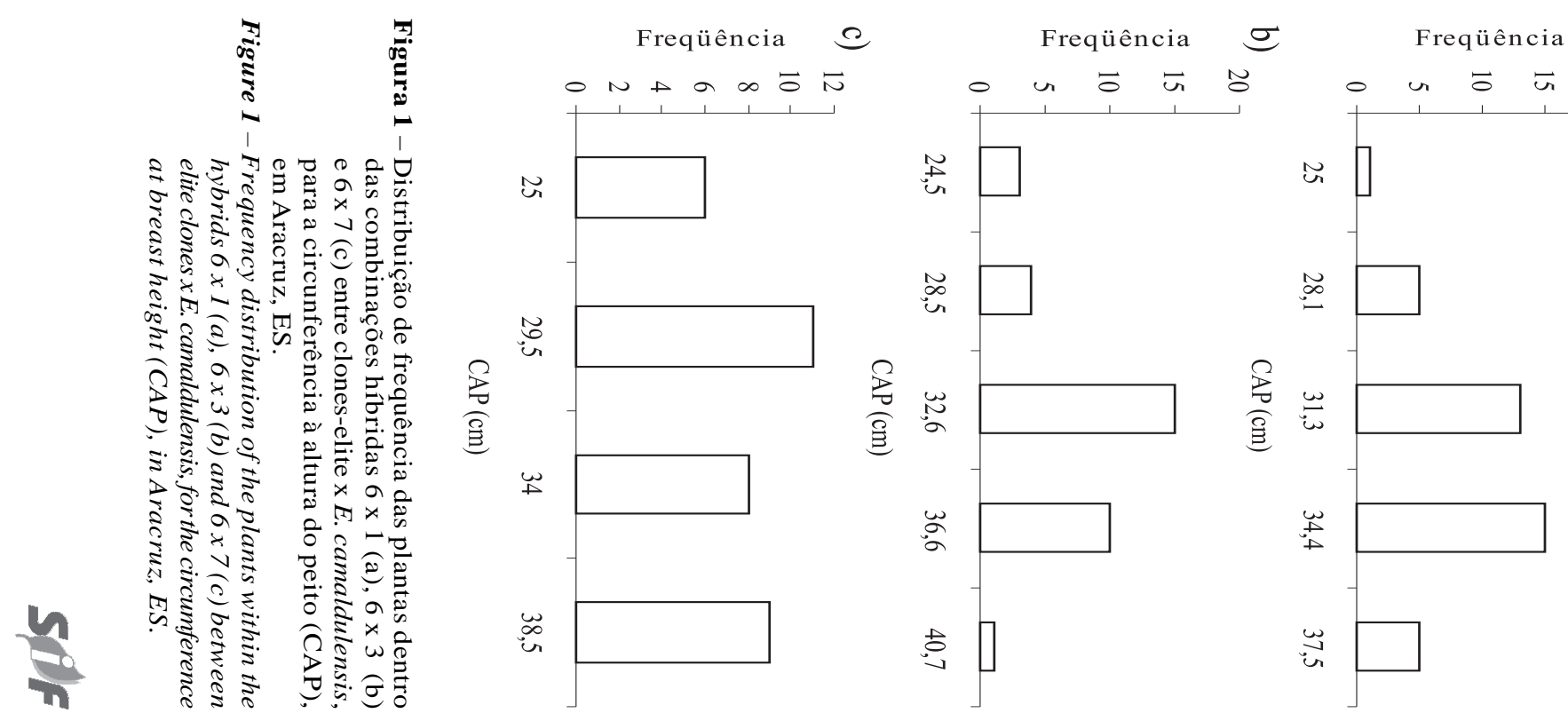

உ

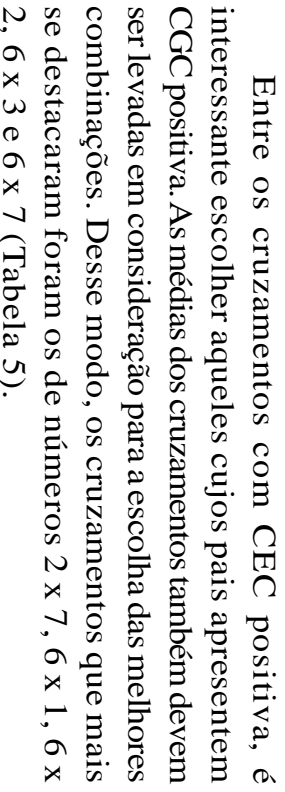


Considerando a DEN, os clones que apresentaram CGC positiva no grupo 1 foram os de números 1,3 e 4 (Tabela 5). No grupo 2, os clones que apresentaram CGC positiva foram os de números 5,6 , e 7 . Nesse grupo, os genitores com CGC positiva também apresentaram CGC positiva para a CAP, evidenciandose que possuem maior frequência de alelos favoráveis, em relação aos demais genitores do grupo 2, quanto às duas características. Os cruzamentos que se destacaram com base na estimativa da CEC e nas médias foram os de números 1 × 10, 3 × 6, 4 × 7 e 4 × 8 (Tabela 5).
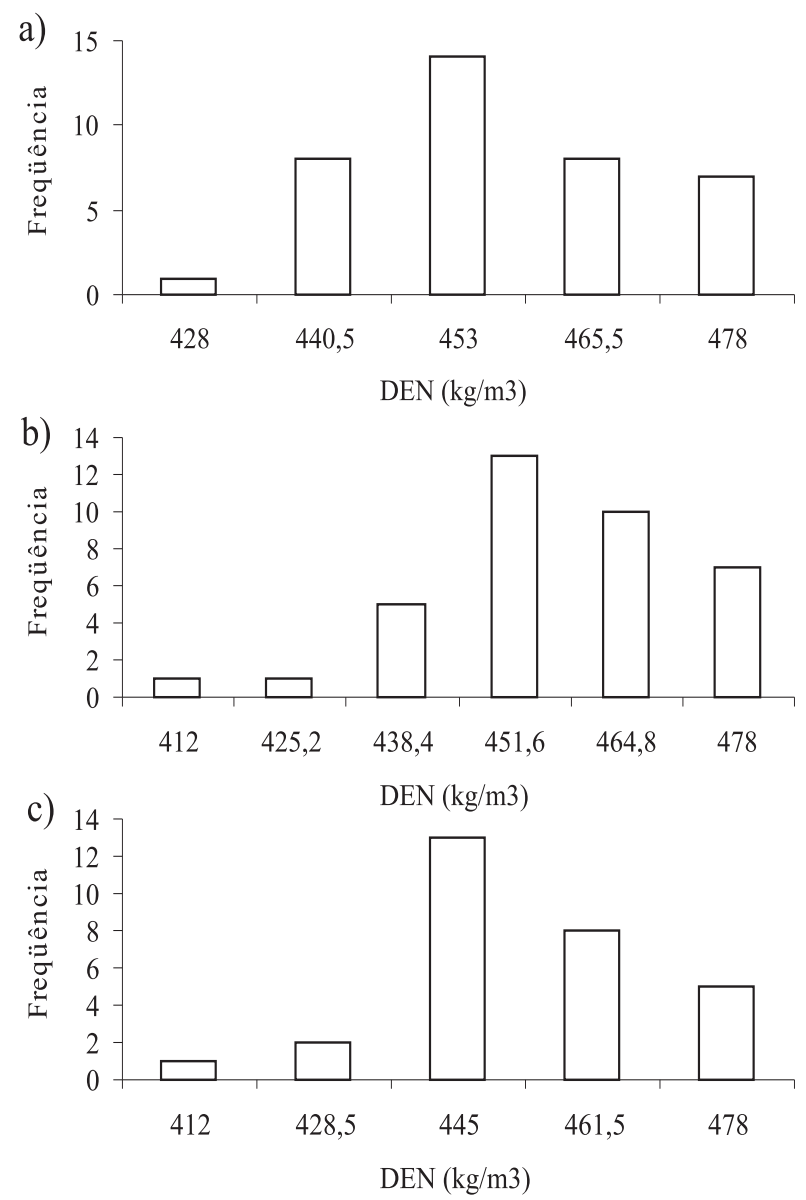

Figura 2 - Distribuição de frequência das plantas dentro das combinações híbridas 1 x 10 (a), 3 × 6 (b) e 4 x 7 (c) entre clones-elite x E. camaldulensis, para a densidade básica da madeira (DEN), em Aracruz, ES.

Figure 2 - Frequency distribution of the plants within the hybrids $1 \times 10(a), 3 \times 6(b)$ and $4 \times 7(c)$ between elite clones $x$ E. camaldulensis, for the basic wood density (DEN), in Aracruz, ES.
No caso de uma espécie em que as informações são obtidas por indivíduo, como no eucalipto, grande vantagem do dialelo é que, ao mesmo tempo que se identifica o cruzamento com maior média, pode-se ter, sem esforço adicional, a estimativa da variabilidade dentro do cruzamento. Desse modo, pode-se identificar a população segregante mais promissora para a seleção, isto é, a que associa média e variância altas. Nas Figuras 1 e 2 são apresentadas as distribuições de frequência da CAP e DEN de alguns cruzamentos que se destacaram no dialelo. Em todos os casos, fica evidenciado que as populações associaram média alta e variabilidade, condições essas necessárias para se ter sucesso com a seleção.

Para a seleção de indivíduos, a alternativa seria a utilização da seleção combinada, conforme preconizado por Resende (2002) e por Bueno Filho e Vencovsky (2000), considerando-se o desempenho de cada indivíduo e também o desempenho da combinação híbrida a que ele pertence.

\section{CONCLUSÕES}

Os cruzamentos envolvendo clones-elite de $E$. grandis, E. urophylla e E. saligna com clones de E. camaldulensis mostraram-se promissores, tanto para a obtenção de ganhos em volume quanto na qualidade da madeira.

A capacidade geral de combinação explicou a maior parte da variação entre os híbridos; assim, a interação alélica aditiva deve ser predominante no controle dos caracteres, especialmente no caso da densidade da madeira.

\section{AGRADECIMENTOS}

Ao CNPq, pela bolsa de estudos; e à empresa Aracruz Celulose S.A., pela oportunidade de desenvolver este trabalho em conjunto com a Universidade Federal de Lavras.

\section{REFERÊNCIAS}

ASSIS, T. F. Production and use of Eucalyptus hybrids for industrial purposes. In: HYBRID BREEDING AND GENETICS OF FOREST TREES, 2000, Noosa. Proceedings... Brisbane: Department of Primary Industries, 2000. p.63-74.

BISON, O. et al. Inbreeding depression in Eucalyptus clones. Crop Breeding and Applied Biotechnology, v.4, p.459-464, 2004.

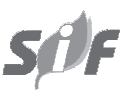

R. Árvore, Viçosa-MG, v.33, n.3, p.395-402, 2009 
BISON, O. et al. Comparison between open pollinated progênies and hybrids performance in Eucalyptus grandis and Eucalyptus urophylla.

Silvae Genetica, v.55, n.1, p.4-5, 2006.

BISON, O. et al. Combining ability of elite clones of Eucalyptus grandis and Eucalyptus urophylla with Eucalyptus globules. Genetics and

Molecular Biology, v.30, n.2, p.417-422, 2007.

BOUVET, J. M.; VIGNERON, P. Variance structure in Eucalyptus hybrid populations. Silvae Genetica, v.45, n.2/3, p.171-177, 1996.

BUENO FILHO, J. S. S.; VENCOVSKY, R. Efficiency of combined selection over sequential selection in forest tree progeny trials. Silvae Genetica, v.49, n.4/5, p.169-173, 2000.

CRUZ, C. D.; REGAZZI, A. J.; CARNEIRO, P. C. S. Modelos biométricos aplicados ao melhoramento genético. 3.ed. Viçosa, MG: Universidade Federal de Viçosa, 2004. 480p.

GERALDI, I. O.; MIRANDA FILHO, J. B. Adapted models for the analysis of combining ability of varieties in partial diallel crosses.

Revista Brasileira de Genética, v.11, n.2, p.419-430, 1988.

GONÇALVES, G. A. et al. Resposta na segunda rotação pela seleção efetuada na primeira, em famílias de meios-irmãos de Eucalyptus grandis Hill. Revista Árvore, v.21, n.3, p.337-384, 1997.

GONÇALVES, F. M. A. et al. Progresso genético por meio da seleção de clones de eucalipto em plantios comerciais. Revista Árvore, v.25, n.3, p.295-301, 2001.
GRIFFING, B. Concept of general and specific combining ability in relation to diallel crossing systems. Australian Journal Biological Science, v.9, p.463-493, 1956.

PAULA, R. C. et al. Estimativas de parâmetros genéticos em famílias de meios-irmãos de Eucalyptus camaldulensis DEHNH, II. Eficiência de utilização de nutrientes. Revista Árvore, v.20, n.4, p.483-493, 1996.

RESENDE, M. D. V. Genética biométrica e estatística: no melhoramento de plantas perenes. Brasília: Embrapa Informação Tecnológica, 2002.975p.

REZENDE, G. D. S. P.; RESENDE, M. D. V. Dominance effects in Eucalyptus grandis, Eucalyptus urophylla and hybrids. In: HYBRID BREEDINGAND GENETICS OF FOREST TREES, 2000, Noosa. Proceedings... Brisbane:

Department of Primary Industries, 2000. p.93-100.

SAS Institute. SAS: user's guide statistical version 8.0. ed. Cary, 2000.

SCOTT, A. J.; KNOTT, M. A. Cluster analyses method for grouping means in the analyses of variance. Biometrics, v.30, n.3, p.507-512, 1974.

VENCOVSKY, R. Herança quantitativa. In: PATERNIANI, E.; VIEGAS, G. D.

Melhoramento e produção do milho. 2.ed. Campinas: Fundação Cargill, 1987. p.135-214.

VENCOVSKY, R.; RAMALHO, M.A.P.

Contribuições do melhoramento genético de plantas no Brasil. In: PATERNIANI, E. (Ed.). Agricultura brasileira e pesquisa agropecuária. Brasília: Embrapa Comunicação para Transferência de Tecnologia, 2000. p.57-89. 